\title{
Neural correlates of socio-emotional perception in 22q11.2 deletion syndrome
}

\author{
Lydia Dubourg $^{1 *}$ (D) Pascal Vrticka ${ }^{3}$, Martin Debbané ${ }^{1,4,5}$, Léa Chambaz ${ }^{1}$, Stephan Eliez ${ }^{1,6}$ and Maude Schneider ${ }^{2}$
}

\begin{abstract}
Background: Social impairments are described as a common feature of the 22q11.2 deletion syndrome (22q11DS). However, the neural correlates underlying these impairments are largely unknown in this population. In this study, we investigated neural substrates of socio-emotional perception.

Methods: We used event-related functional magnetic resonance imaging (fMRI) to explore neural activity in individuals with 22q11DS and healthy controls during the visualization of stimuli varying in social (social or non-social) or emotional (positive or negative valence) content.

Results: Neural hyporesponsiveness in regions of the default mode network (inferior parietal lobule, precuneus, posterior and anterior cingulate cortex and frontal regions) in response to social versus non-social images was found in the 22q11DS population compared to controls. A similar pattern of activation for positive and negative emotional processing was observed in the two groups. No correlation between neural activation and social functioning was observed in patients with the 22q11DS. Finally, no social $\times$ valence interaction impairment was found in patients.

Conclusions: Our results indicate atypical neural correlates of social perception in 22q11DS that appear to be independent of valence processing. Abnormalities in the social perception network may lead to social impairments observed in 22q11DS individuals.
\end{abstract}

Keywords: 22q11.2 deletion syndrome, Socio-emotional perception, Default mode network, fMRI

\section{Background}

The 22q11.2 deletion syndrome (22q11DS), also known as DiGeorge or velocardiofacial syndrome (VCFS), is a neurogenetic disorder affecting approximately 1 in 4000 live births [1] and occurring in up to 1:1000 pregnancies [2]. The syndrome is associated with elevated risk for schizophrenia [3] and is characterized by high rates of distinct positive and negative symptoms. It is well established that social dysfunction is also a common feature of the 22q11DS profile [4-6]. Individuals with 22q11DS are described as shy, withdrawn, and presenting social interaction problems particularly with their peers [4-6]. Up until now, only a few neuroimaging studies have investigated the neural correlates of socio-cognitive processes in the 22q11DS population to better understand the emergence of social deficits associated with the

\footnotetext{
* Correspondence: lydia.dubourg@unige.ch

${ }^{1}$ Developmental Imaging and Psychopathology Laboratory, Department of Psychiatry, School of Medicine, University of Geneva, Campus Biotech, Chemin des mines 9, 1202 Geneva, Switzerland

Full list of author information is available at the end of the article
}

syndrome [7-9]. The neural substrates of social-emotional impairments hence remain largely unclear. In the current study, we therefore focused on the neural correlates of socio-emotional processes to extend our understanding of impairments in the 22q11DS population related to such mechanisms.

Social perception encompasses the processing of various social cues that individuals encounter in everyday life (e.g. face and voice). Social cue perception has been mostly investigated through face perception. In healthy individuals, face perception is mainly associated with increased activity in the fusiform face area, visual extrastriate cortex, lateral occipital gyri, anterior temporal pole, and posterior superior temporal gyrus $[10,11]$. Moreover, the processing of emotional information in faces involves a similar network as emotion perception (see below), including limbic regions, inferior frontal gyrus, medial prefrontal gyrus, and putamen [11-13].

Until recently, most studies in patients with schizophrenia focused on higher order processes of social 
cognition (e.g. theory of mind) while lower aspects as social perception have received less attention. To our knowledge, only one study has examined brain activations in response to simple visual social cues [14]. This study reported abnormal neural activity during processing of social information. Hypoactivation in regions associated with visual processing (occipital and temporal regions) and increased cingulate activity during the processing of social vs. non-social images has been observed in patients with schizophrenia compared to controls. In patients with 22q11DS, as in schizophrenia, most studies have focused on higher-order socio-cognitive processes, highlighting impairments in theory of mind and emotion recognition [15, 16], and few functional magnetic resonance imaging (fMRI) studies have been conducted on this topic [7-9]. Andersson et al. [8] investigated social perception and reported hypoactivation of the fusiform in response to faces versus houses. In light of the high prevalence of social impairments in 22q11DS, additional studies examining the neural substrates of social perception and their link with social functioning are clearly needed.

A second aspect of social cognition that will be investigated in this study is emotion processing. Commonly, these processes are investigated while individuals are viewing pleasant or unpleasant images compared to neutral images. In healthy participants, a set of regions has been related to emotion processing, mainly including the limbic system (amygdala, anterior hippocampus, anterior insula, and cingulate gyrus), as well as brain stem nuclei, thalamus, ventral striatum, medial prefrontal cortex, posterior cingulate cortex, precuneus, lateral temporal cortex, and temporal pole [17, 18]. However, up until now, only three studies have investigated the neural basis of emotion processing in 22q11DS [7-9]. van Amelsvoort et al. [7] compared brain activity during the presentation of mixed emotional facial expressions between eight individuals with 22q11DS and nine controls. Two types of facial emotions (happy or angry) and neutral faces were presented in a block design. Patients with 22q11DS showed less activation in the right insula and frontal regions and more activation in occipital regions compared to controls. However, this study used a block design (with mixed emotion), which made it impossible to differentiate neural responses as a function of the type of valence. Two additional studies used only negatively valenced stimuli $[8,9]$ and confirmed the hypoactivation in regions related to emotion processing (superomedial prefrontal cortices) in patients with 22q11DS. However, and contrary to van Amelsvoort et al. [7], some regions involved in socio-cognitive processing (fusiform, anterior cingulate cortex) were also hypoactivated. Moreover, only one study investigated the relationship between brain activation and social functioning in individuals with 22q11DS [9]. The authors found that decreased brain activation during emotion perception was related to social difficulties in patients with 22q11DS. So far, studies investigating emotional processing in participants with 22q11DS reported results from very small samples (8 to 15 patients) and only one type of valence was explored. Consequently, the neural bases of emotion processing still remain unclear in this population, and the distinction between positive and negative emotion perception has never been properly investigated.

Finally, it is important to take into consideration that emotions can influence social processing, as it appears that there is an overlap between social information and emotion processing. Indeed, it has been argued that social cues are inherently emotional and that social information processing will therefore also involve emotionprocessing networks [19]. To test this hypothesis, several studies in healthy individuals have been conducted and confirmed an interactive processing of social content and valence [19-21]. While a social content $\times$ valence interaction effect was found in the thalamus, superior temporal sulcus, middle orbito-temporal cortex, as well as in the anterior insula and lateral medial prefrontal cortex in several studies, Norris et al., Scharpf et al., and Vrtička et al. [19-21] also observed such interaction effect in the amygdala, fusiform gyrus, anterior superior frontal gyrus, and middle occipital cortex. Conversely, in the 22q11DS, the influence of valence on social and non-social information processing is currently unknown.

This study examined neural correlates of socioemotional processing patients with 22q11DS compared to healthy controls. First, we investigated neural correlates of social perception. In line with the literature in schizophrenia [14], we expected to observe altered activations in the social perception network in participants with 22q11DS. Secondly, we examined neural response to positively and negatively valenced stimuli. Based on previous studies in participants with 22q11DS, we expected to observe a significant decrease in regions involved in emotion (e.g. insula, frontal regions) and socio-cognitive processing (e.g. fusiform). Thirdly, in order to investigate the influence of emotions on social perception in 22q11DS, we also examined a social content $x$ valence interaction. Finally, we hypothesized that impaired activation of socio-emotional networks would be related to socio-cognitive deficits in participants with 22q11DS.

\section{Methods}

\section{Participants}

Participants were recruited through parent associations or word of mouth and were tested in our research laboratory during an ongoing longitudinal study. Twentytwo participants with 22q11DS aged between 12 and 32 
were included (mean age $=20.3 \pm 5,17$ (77\%) females). The presence of a 22q11.2 microdeletion was confirmed in all participants using quantitative fluorescent polymerase chain reaction (QF-PCR). Patients diagnosed with a DSM-IV psychotic disorder were excluded from this study $(N=2)$ to decrease the influence of confounding factors on brain activation patterns (e.g. long-term use of antipsychotics). However, some patients met formal diagnostic criteria for other current psychiatric conditions, and 10 participants were under medication at the time of testing (see Table 1). Furthermore, 22 controls including siblings $(n=13)$ and unrelated individuals $(N=9)$ aged between 12 and 32 (mean age $=19.7 \pm 5,15$ (68\%) females) were also included and were screened for the presence of any neurological problems, and psychological or learning difficulties (see Table 1).

Written informed consent was obtained from participants and their parents under protocols approved by the Swiss Ethics Committee on research involving humans.

\section{Clinical assessment}

The presence of psychiatric disorders was assessed in adolescents below 18 years using the Diagnostic Interview for Children and Adolescents - Revised [22], and the mood and psychosis supplement of the Kiddie-Schedule for Affective Disorders and Schizophrenia Present and Lifetime version (K-SADS-PL; [23]). Adult participants were screened using the Structured Clinical Interview for DSM-IV axis disorders (SCID-I; [24]). Participants were also screened for attenuated positive and negative symptoms using the Structured Interview for Psychosisrisk Syndromes (SIPS; [25]). Symptoms are assessed on a 7-point severity scale (ranging from 0 to 6).

\section{Intellectual functioning}

All participants completed the Wechsler Intelligence Scale for Children III or IV (WISC-III-R or WISC-IV-R; [26, 27]) or Adult III or IV (WAIS III or WAIS IV; $[28,29]$ ) in order to obtain an evaluation of global intellectual functioning. Mean full-scale IQ was $74(\mathrm{SD}=12)$ in participants with 22q11DS and $118(\mathrm{SD}=11)$ in controls.

Table 1 Demographic informations for 22q11DS and healthy control participants

\begin{tabular}{|c|c|c|c|c|c|c|}
\hline & & & \multicolumn{2}{|c|}{ Diagnostic group } & \multicolumn{2}{|c|}{ Comparison } \\
\hline & & & $22 q 11 D S$ & Controls & ANOVA & $\overline{p \text { value }}$ \\
\hline \multicolumn{3}{|l|}{$\bar{N}$} & 22 & 22 & & \\
\hline \multicolumn{3}{|l|}{ Age } & $20.3( \pm 5.2)$ & $19.7( \pm 5.1)$ & 0.120 & 0.730 \\
\hline \multicolumn{3}{|l|}{ Gender (\% of female) } & $17(77 \%)$ & $15(68 \%)$ & 0.442 & 0.510 \\
\hline \multirow{2}{*}{\multicolumn{3}{|c|}{$\begin{array}{l}\text { Full IQ (mean (SD)) Benton } \\
\text { face recognition }\end{array}$}} & $74( \pm 12)$ & $118( \pm 11)$ & 162.8 & $<0.01$ \\
\hline & & & $39.4(3.9)$ & $47.6(2.9)$ & 60.1 & 0.001 \\
\hline \multirow[t]{6}{*}{ SRS $(\text { mean }(S D))^{*}$} & & SRS awareness & $58( \pm 12)$ & $47( \pm 9)$ & 7.4 & 0.001 \\
\hline & & SRS cognition & $57( \pm 9)$ & $45( \pm 6)$ & 17.4 & $<0.001$ \\
\hline & & SRS communication & $58( \pm 11)$ & $45( \pm 5)$ & 20.4 & $<0.001$ \\
\hline & & SRS motivation & $60( \pm 12)$ & $44( \pm 6)$ & 18.1 & $<0.001$ \\
\hline & & SRS RRB & $58( \pm 11)$ & $45( \pm 5)$ & 20.4 & $<0.001$ \\
\hline & & SRS Total & $51( \pm 12)$ & $44( \pm 6)$ & 18.1 & $<0.001$ \\
\hline \multirow[t]{9}{*}{ Psychiatric diagnosis (N (\%)) } & & Major depression disorder & $5(23 \%)$ & & & \\
\hline & & Specific phobia & $3(13 \%)$ & & & \\
\hline & & Simple phobia & $1(4 \%)$ & & & \\
\hline & & Generalized anxiety disorder & $3(13 \%)$ & & & \\
\hline & & Obsessive compulsive disorder & $1(4 \%)$ & & & \\
\hline & & Alcohol dependence & $1(4 \%)$ & & & \\
\hline & & Oppositional defiant disorder & $1(4 \%)$ & & & \\
\hline & & Delusions & $2(8 \%)$ & & & \\
\hline & & ADHD & $3(13 \%)$ & & & \\
\hline \multirow[t]{3}{*}{ Psychotropic medication } & Categories & Antipsychotics & $1(4 \%)$ & & & \\
\hline & & Antidepressants & $7(32 \%)$ & & & \\
\hline & & Methylphenidate & $4(18 \%)$ & & & \\
\hline
\end{tabular}

*For the SRS, data were missing for five 22q11DS participants and six controls 


\section{Socio-cognitive measures}

We also assessed socio-cognitive functioning in all participants. To do so, we administered the Benton Facial Recognition Test (BFRT; [30]), a measure of face recognition ability. Participants were asked to match nonemotional unfamiliar faces. They were presented one target and six other black and white faces (male or female). First, participants were asked to match the target face with an identical photo. Secondly, they had to match the target face with three photos taken from different angles or different lighting conditions. The total number of correct answer was used as main measure. Scores ranging from 41 to 54 indicate normal performance, from 39 to 40 borderline performance, between 37 and 38 moderate impairment, and below 37 severe impairment.

We also administered the second edition of the Social Responsiveness Scale (SRS-2; [31]), a measure of social functioning to parents of 17 participants with the 22q11DS and 16 controls to identify the presence and severity of social impairments. Data were missing for 11 individuals. The SRS is a 65 -item parent questionnaire investigating the child's social behaviour in the past 6 months. A 4-point Likert scale $(0=$ not true, $1=$ sometimes true, 2 = often true, 3 = always almost true) is used to rate how often the child engages in the respective behaviour. The SRS provides information about five domains: social awareness, social cognition, social communication, social motivation, and restricted interests and repetitive behaviour. Raw scores for each scale are converted to a gender-specific T score representing the individual's social behaviour impairment in each of the five domains. The five scales are summed and converted into a $\mathrm{T}$ score, resulting in an overall composite SRS score. T scores up to 59 are within the normal range, from 60 to 65 indicate mild deficiencies of reciprocal social behaviour, from 66 to 75 moderate impairment, and $\geq 76$ severe impairment.

\section{fMRI paradigm: design and procedure}

The experiment consisted of two runs during which participants were presented with images from the International Affective Picture System (IAPS; [32]) and scrambled images. The scrambled images were created using the selected IAPS pictures (obtained using the Photoshop plugin: [33]. Participants were instructed to indicate whether the image was intact or scrambled using an MRI-compatible response box. Target images were divided into four categories using a 2 (social content) $\times 2$ (valence) factorial design: social and positive valence, social and negative valence, non-social and positive valence, and non-social and negative valence. Positive and negative images were selected to elicit similar arousal levels (see Additional file 1: Table S1). Social images were defined as pictures that contained at least two human beings, while non-social images did not include any humans. During each run, 40 images (10 from each category) and 40 scrambled images were presented for $2 \mathrm{~s}$ with inter-trial intervals varying between 2500 and $5000 \mathrm{~ms}$ (Fig. 1). The presentation order of the two runs was counterbalanced between participants.

\section{Behavioural analysis}

Between- and within-group comparisons of reaction time in response to the different conditions (social, nonsocial, positive, negative, scrambled) as well as accuracy were examined using univariate ANOVAs in SPSS Version 21.

\section{fMRI data acquisition and analysis}

Structural and functional images were acquired using a Siemens Prisma $3 \mathrm{~T}$ scanner at the Geneva Center for Biomedical Imaging (CIBM). The acquisition protocol for the structural sequence was a $3 \mathrm{D}$ volumetric pulse sequence with $\mathrm{TR}=2500 \mathrm{~ms}$, TE $=3 \mathrm{~ms}$, flip angle $=8^{\circ}$,
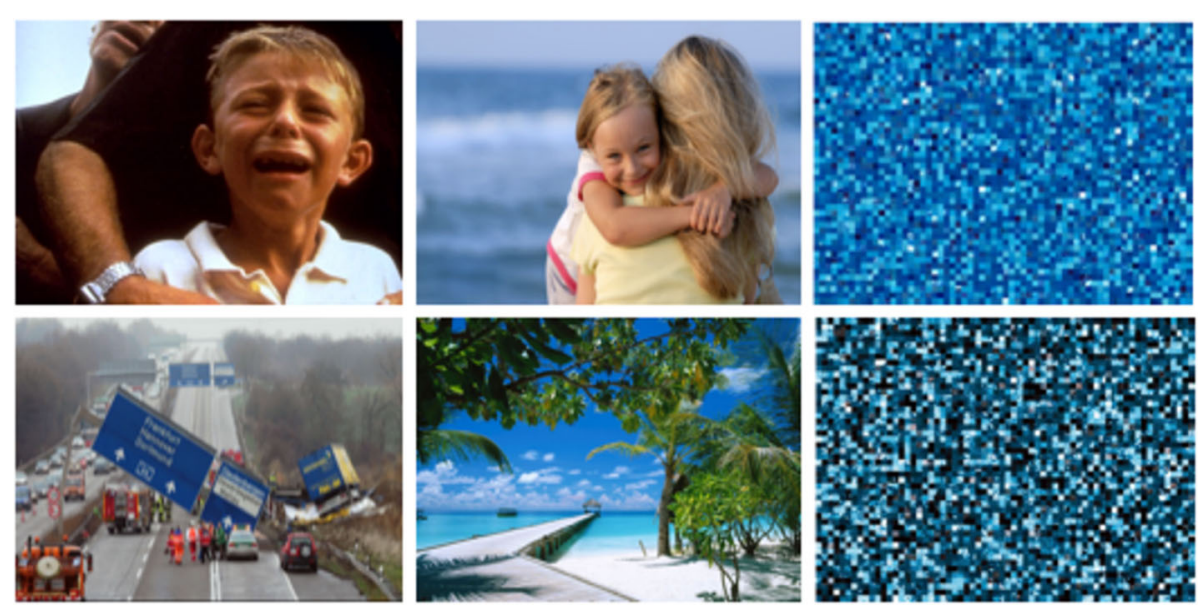

Fig. 1 Example of stimuli. Left of right: negative, positive, and blurred. Top panel: social, bottom panel: non-social 
acquisition matrix $=256 \times 256$, field of view $=22 \mathrm{~cm}$, slice thickness $=1.1 \mathrm{~mm}$, and 192 slices. The fMRI acquisition consisted of two sequences of $8 \mathrm{~min}$ each and resulting in 430 blood-oxygenation-level-dependent (BOLD) images $(\mathrm{TR}=2200 \mathrm{~ms}$, echo time, $\mathrm{TE}=30 \mathrm{~ms}$, 36 axial slices, slice thickness $=4.0 \mathrm{~mm}$, spacing between slices $=2.5 \mathrm{~mm}$, flip angle $=85^{\circ}$, field of view $[\mathrm{FOV}]=235 \mathrm{~mm}$ ).

fMRI data were processed and analysed using Statistical Parametric Mapping 12 (SPM12; Welcome Department of Neuroscience, London UK). Functional images were realigned using rigid body registration and resliced. Participants with motion exceeding $3 \mathrm{~mm}$ in any of the six directions were excluded from the analyses (four patients and two controls).

Each participant's structural image was coregistered to the mean of the realigned functional images and segmented with the Dartel option to obtain tissue classification. Finally, we normalized to $1 \mathrm{~mm}^{3}$ Montreal Neurologic Institute (MNI) space and spatially smoothed with a 6-mm at fullwidth half-maximum three-dimensional Gaussian kernel, employing the diffeomorphic anatomical registration using exponential lie algebra algorithm (DARTEL).

For each participant, brain responses were analysed in the context of the general linear model (GLM) approach using a two-level procedure. At the first level, the five experimental conditions (social positive, social negative, non-social positive, non-social negative, and scrambled) were entered in the model. The six movement parameters were included as additional regressors of no interest in the design matrix. Changes in the BOLD signal were obtained using the estimated GLM parameters for each contrast of interest (social vs. non-social, social vs. scrambled, non-social vs. scrambled, positive vs. negative, positive vs. scrambled, negative vs. scrambled) and interaction contrasts (social content $\times$ valence). In the second-level analysis, we first computed the overall effect per group for each contrast; then, individual contrast images were included in an independent two-sample $t$-test to determine significant brain activation in patients with 22q11DS versus healthy controls. In order to account for the possible influence of age, sex, IQ, and handedness on brain activation, these four variables were entered as control covariates in the model for the second-level analysis (see Additional file 1 for a description of the results without IQ as covariate). Moreover, as medication could also be an important confound in this study, the presence or absence of medication was added as covariate in all the analyses.

For the within- and between-group comparisons, we chose a primary voxel-level statistical threshold of $p<0.001$ (uncorrected, whole brain) and $k \geq 20$. For clusters that survived this threshold at the voxel level, a cluster-extent family-wise correction (FWEc) for multiple comparisons at $p<0.05$ was applied. Neuroanatomical locations of activations were identified using Talairach Daemon software after adjusting coordinates to allow differences between the MNI and Talairach templates [34].

To investigate potential associations between brain activity differences of the main contrasts and clinical variables, we first extracted raw activations (betas) from significant clusters and a priori ROIs (see below). Then, using multiple regression analyses, we tested these betas against face recognition and social functioning scores with age, gender, handedness, and IQ as covariates. Secondly, we performed whole-brain multiple regression analyses. Each regression included one contrast (i.e. social vs. non-social), included the covariates of interest (socio-cognitive measures), and was controlled for age, sex, handedness, and IQ. Effects of covariates on brain activations were investigated in both directions. A combined statistical threshold of $p<0.001$ uncorrected at the peak and $p<0.05$ FWE-corrected at the cluster level was applied.

\section{Results}

\section{Behavioural results}

Reaction time values are provided in Table 2. Within both groups, reaction times were faster for scrambled versus intact images $(p<.05)$. However, no significant differences were present for intact images regarding social content (social/non-social) and valence (positive/ negative) $(p>.05)$. Conversely, between-group comparisons revealed that 22q11DS participants had significantly faster reaction times compared to controls in all experimental conditions except for scrambled images $(p<.05)$. Comparison of accuracy within and between groups did not reveal any significant differences $(p>0.05)$.

\section{Neuroimaging results \\ Social perception}

Social versus scrambled contrast We first determined brain activation in response to social stimuli (social vs. scrambled trials). In the control group, this contrast revealed enhanced activation of the left postcentral gyrus and inferior parietal lobule (see Table 3). In participants with 22q11DS, the same contrast did not show any

Table 2 Reaction times for 22q11DS (22q) and healthy control (HC) participants

\begin{tabular}{lll}
\hline Condition & \multicolumn{2}{l}{ Reaction times $(\mathrm{ms})$} \\
\cline { 2 - 3 } & 22q $(\mathrm{M} \pm \mathrm{SD})$ & $\mathrm{HC}(\mathrm{M} \pm \mathrm{SD})$ \\
\hline Social & $818.4 \pm 178.2$ & $834.8 \pm 178.7$ \\
Non-social & $846.3 \pm 147.5$ & $945.43 \pm 551.5$ \\
Positive & $833.1 \pm 153.6$ & $926.5 \pm 551.7$ \\
Negative & $831.5 \pm 171.2$ & $853 \pm 189.1$ \\
Scrambled & $711.9 \pm 129.6$ & $700.55 \pm 130.6$ \\
\hline
\end{tabular}


Table 3 Brain regions showing significant increase in social perception contrasts within and between groups

\begin{tabular}{|c|c|c|c|c|c|c|c|}
\hline \multirow{2}{*}{\multicolumn{2}{|c|}{$\frac{k}{\text { Healthy controls }}$}} & \multicolumn{3}{|c|}{ MNI $(x, y, z)$} & \multirow[t]{2}{*}{ Hemisphere } & \multirow[t]{2}{*}{ Region } & \multirow[t]{2}{*}{ BA } \\
\hline & & & & & & & \\
\hline \multicolumn{8}{|c|}{ Social > Scrambled } \\
\hline \multirow[t]{7}{*}{1095} & 5.36 & -40 & -39 & 49 & L & Inferior parietal lobule & 40 \\
\hline & 5.26 & -47 & -34 & 50 & $\mathrm{~L}$ & Postcentral gyrus & 2 \\
\hline & 4.43 & -52 & -25 & 50 & $\mathrm{~L}$ & Postcentral gyrus & 2 \\
\hline & 4.35 & -50 & -47 & 56 & $\mathrm{~L}$ & Inferior parietal lobule & 40 \\
\hline & 4.17 & -47 & -42 & 56 & L & Inferior parietal lobule & 40 \\
\hline & 4.16 & -41 & -47 & 56 & L & Inferior parietal lobule & 40 \\
\hline & 3.69 & -53 & -22 & 53 & L & Postcentral gyrus & \\
\hline \multicolumn{8}{|c|}{ Nonsocial > Scrambled } \\
\hline \multirow[t]{3}{*}{4239} & 5.92 & -13 & -103 & 8 & L & Cuneus & 18 \\
\hline & 5.34 & -6 & -100 & 14 & L & Middle occipital gyrus & 18 \\
\hline & 5.33 & -4 & -94 & 23 & L & Cuneus & 19 \\
\hline \multirow[t]{3}{*}{1678} & 6.00 & -26 & -71 & -7 & $\mathrm{~L}$ & Lingual gyrus & 19 \\
\hline & & -22 & -73 & -14 & L & Lingual gyrus & 18 \\
\hline & & -9 & -83 & -11 & $\mathrm{~L}$ & Lingual gyrus & 18 \\
\hline \multirow[t]{3}{*}{1244} & 7.86 & 12 & -97 & 18 & $\mathrm{R}$ & Cuneus & 17 \\
\hline & 5.65 & 13 & -89 & -7 & $\mathrm{R}$ & Lingual gyrus & 17 \\
\hline & 4.76 & 16 & -96 & -2 & $\mathrm{R}$ & Cuneus & 17 \\
\hline \multirow[t]{2}{*}{622} & 6.10 & 21 & -63 & 13 & $\mathrm{R}$ & Posterior cingulate & 31 \\
\hline & & 17 & -62 & 4 & $\mathrm{R}$ & Posterior cingulate & 30 \\
\hline
\end{tabular}

Social $>$ Non-Social No cluster pFWEc $<0.05$

22a11DS patients

Social $>$ Scrambled No cluster pFWEc $<0.05$

Nonsocial > Scrambled

$\begin{array}{llllll}1416 & 6.45 & -13 & -91 & 8 & L \\ 5.70 & -5 & -97 & 7 & L \\ 5.68 & -11 & -96 & 5 & L \\ 5.26 & -9 & -100 & 12 & L \\ & 4.65 & -17 & -93 & 17 & L \\ & 4.35 & -13 & -95 & 17 & L \\ & 5.62 & -28 & -41 & 72 & L \\ & 4.96 & -22 & -40 & 73 & L \\ & 4.53 & -26 & -39 & 62 & L\end{array}$

Social $>$ Non-Social No cluster pFWEc $<0.05$

Group comparison

Social $>$ Scrambled No cluster pFWEc $<0.05$

Non-social $>$ Scrambled No cluster pFWEc $<0.05$

Social > Non-Social CTRL > VCFS

$\begin{array}{ccc}5.14 & 0 \\ 4.93 & -11 \\ 4.26 & -11 \\ 4.22 & -19 \\ 4.18 & -11\end{array}$

$\begin{array}{ll}46 & 20 \\ 51 & 14 \\ 39 & 18 \\ 41 & 15 \\ 43 & 19\end{array}$

Medial frontal gyrus 9

Medial frontal gyrus $\quad 10$

Anterior cingulate $\quad 32$

Anterior cingulate $\quad 32$

Medial frontal gyrus 9

$\begin{array}{ll}\text { Cuneus } & 17 \\ \text { Cuneus } & 18 \\ \text { Cuneus } & 17 \\ \text { Middle occipital gyrus } & 17 \\ \text { Middle occipital gyrus } & 18 \\ \text { Cuneus } & 18 \\ \text { Postcentral gyrus } & 2 \\ \text { Postcentral gyrus } & 2 \\ \text { Postcentral gyrus } & 3\end{array}$


Table 3 Brain regions showing significant increase in social perception contrasts within and between groups (Continued)

\begin{tabular}{|c|c|c|c|c|c|c|c|}
\hline k & $t$ & $\mathrm{MNI}$ & & & Hemisphere & Region & $\mathrm{BA}$ \\
\hline & 4.11 & -7 & 46 & 21 & $L$ & Medial frontal gyrus & 9 \\
\hline & 4.09 & -13 & 45 & 9 & L & Anterior cingulate & 32 \\
\hline & 3.92 & -5 & 50 & 30 & $L$ & Medial frontal gyrus & 9 \\
\hline & 3.77 & -11 & 41 & 11 & L & Anterior cingulate & 32 \\
\hline & 3.72 & -6 & 45 & 11 & $L$ & Anterior cingulate & 32 \\
\hline & 3.41 & -7 & 53 & 28 & L & Superior frontal gyrus & 9 \\
\hline 1466 & 5.71 & 52 & -61 & 39 & $\mathrm{R}$ & Inferior parietal lobule & 40 \\
\hline & 5.05 & 52 & -57 & 38 & $\mathrm{R}$ & Inferior parietal lobule & 40 \\
\hline & 4.63 & 47 & -58 & 35 & $\mathrm{R}$ & Supramarginal gyrus & 40 \\
\hline 908 & 4.80 & -26 & 27 & 41 & $L$ & Middle frontal gyrus & 8 \\
\hline & 4.75 & -25 & 29 & 45 & $L$ & Middle frontal gyrus & 8 \\
\hline & 3.98 & -33 & 25 & 41 & L & Middle frontal gyrus & 8 \\
\hline & 3.97 & -16 & 34 & 45 & L & Superior frontal gyrus & 8 \\
\hline & 3.74 & -25 & 43 & 34 & $L$ & Superior frontal gyrus & 9 \\
\hline & 3.66 & -21 & 37 & 43 & $L$ & Superior frontal gyrus & 8 \\
\hline & 3.53 & -25 & 41 & 38 & L & Middle frontal gyrus & 9 \\
\hline 659 & 4.37 & 42 & 20 & 40 & $\mathrm{R}$ & Precuneus & 9 \\
\hline & 4.23 & 41 & 20 & 44 & $\mathrm{R}$ & Middle frontal gyrus & 8 \\
\hline & 4.16 & 34 & 23 & 49 & $\mathrm{R}$ & Middle frontal gyrus & 6 \\
\hline & 3.82 & 34 & 2 & 39 & $\mathrm{R}$ & Middle frontal gyrus & 6 \\
\hline & 3.79 & 29 & 20 & 37 & $\mathrm{R}$ & Middle frontal gyrus & 9 \\
\hline & 3.54 & 36 & 26 & 43 & $\mathrm{R}$ & Middle frontal gyrus & 8 \\
\hline 551 & 4.67 & 13 & -43 & 34 & $\mathrm{R}$ & Posterior cingulate & 31 \\
\hline
\end{tabular}

Abbreviations: $k$ cluster size, $t$ t scores, MNI Montreal Neurological Institute, BA Brodman Area

significant activation, and the between-group comparison did not return any significant difference either.

Non-social versus scrambled contrasts Similar analyses were performed to determine regions activated during non-social image perception. Separate contrasts in each group revealed that controls showed significant activation to non-social images in bilateral cuneus, lingual gyrus, left middle occipital gyrus, and right posterior cingulate, whereas in participants with 22q11DS, non-social images significantly activated the left cuneus, middle occipital gyrus, and left postcentral gyrus. Finally, direct between-group comparison did not reveal any significant difference.

Social versus non-social contrast Next, we tested brain activation specifically associated with social perception (social vs. non-social images). No significant pattern of activation was observed in the within-group comparisons. However, the direct between-group comparison showed greater activation in controls compared to individuals with 22q11DS in the bilateral medial frontal gyrus, left anterior cingulate, middle frontal gyrus, and superior frontal gyrus, as well as in the right supramarginal gyrus, inferior parietal lobule, precuneus, and posterior cingulate (see Fig. 2 and Table 3).

In previous studies conducted in healthy controls using similar images, the same contrast (social > non-social images) elicited a much wider network of activation, notably including the bilateral fusiform gyrus, bilateral amygdala, and superior temporal sulcus [35]. We consequently conducted a post hoc analysis for these regions of interest. Using Marsbar [36], spheres with a 6 - $\mathrm{mm}$ radius were defined around the center of these regions of interest bilaterally. Beta values per individual and per condition were extracted, averaged, and used for ANOVAs and $t$-tests in SPSS. No significant differences within or between groups for these three ROIs were observed.

\section{Emotion processing}

Positive emotional processing To investigate brain activation related to the processing of positive emotional stimuli, we then contrasted positive vs. scrambled images. The within-group contrast revealed lingual gyrus activation in both groups. Conversely, increased activation to 


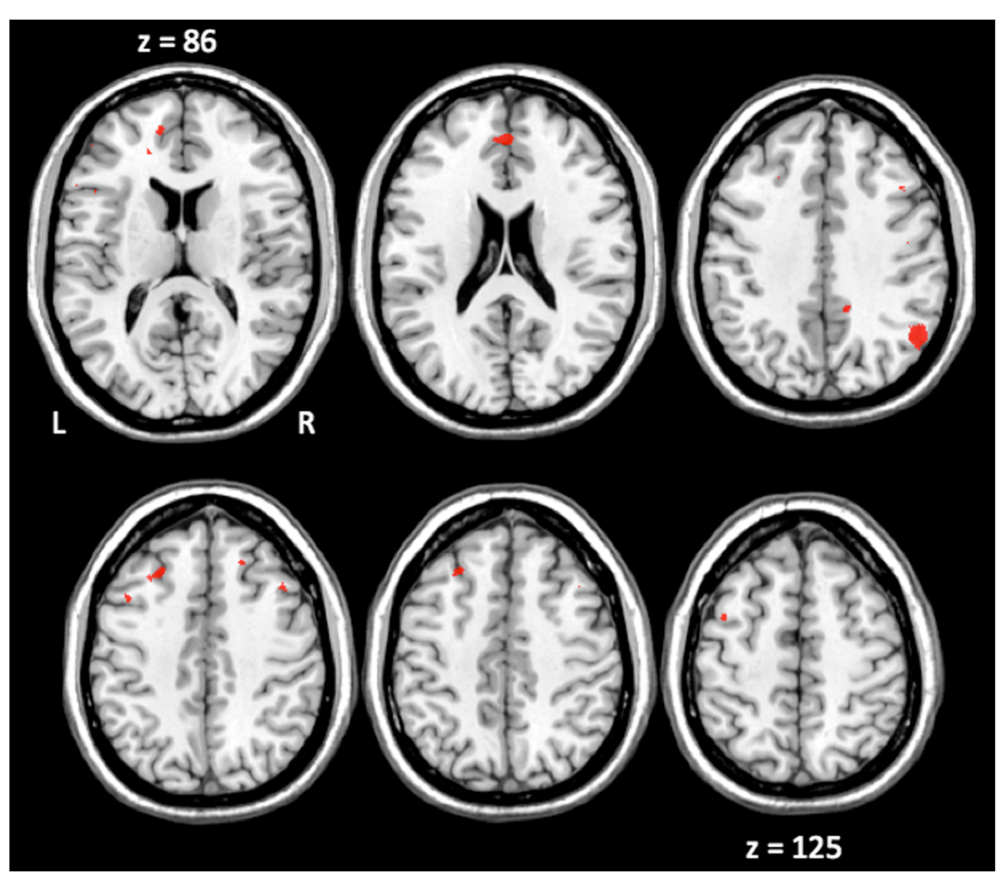

Fig. 2 Increased activation in healthy participants compared to patients with 22q11DS for the condition Social > Non-social. Activations are reported in Table 3. ( $L=$ left, $R=$ right)

positive images was found in the posterior cingulate and cuneus only in healthy controls, while increased activation to positive images was observed in the precuneus, postcentral gyrus, and superior and inferior parietal lobule only in patients (see Table 4). Nonetheless, the direct between-group comparison did not show any significant differences between controls and 22q11DS participants.

Negative emotion processing Brain activation to negative emotional images was subsequently tested (negative vs. scrambled images). In controls, greater activation to negative images was observed in the right lingual gyrus, bilateral cuneus, and left posterior cingulate. No significant activation was present in participants with 22q11DS, and the direct between-group comparison did not reveal any difference between controls and individuals with 22q11DS, either (see Table 4).

Positive versus negative emotion processing Finally, we contrasted positive vs. negative emotional stimuli to determine regions activated as a function of stimulus valence. Both within- and between-group comparisons did not reveal any significant results.

\section{Social content $\times$ valence interaction}

The social $\times$ valence content interaction was tested within and between groups. No significant activations were found.

\section{Association with socio-cognitive measures}

Finally, we were interested in assessing whether there were any associations between brain activity and sociocognitive measures in participants with 22q11DS. To do so, we first extracted raw activations (betas) from significant clusters showing up in the analyses (plus a priori ROIs) and tested these betas against face recognition and social functioning scores. No significant associations were observed. Second, we conducted wholebrain multiple-regression analyses with face recognition and social functioning scores. Again, correlations did not reveal any significant effects.

\section{Discussion}

The present study aimed at identifying the neural correlates of socio-emotional perception in individuals with 22q11DS. Results indicated neural hyporesponsiveness within the social perception network in participants with 22q11DS compared to controls. Second, comparable to healthy controls, individuals with 22q11DS showed activation in regions related with emotion processing during the presentation of positive and negative stimuli. No correlation between brain activation and the clinical measures was observed in the 22q11DS population. Finally, results showed no social content $x$ valence interaction differences between patients and controls.

This is the first study investigating the neural correlates of complex social stimuli perception in patients with 22q11DS. In line with our hypothesis, participants 
Table 4 Brain regions showing significant increase in emotional experience contrasts within and between groups

\begin{tabular}{|c|c|c|c|c|c|c|c|}
\hline k & $t$ & $\mathrm{MNI}$ & & & Hemisphere & Region & BA \\
\hline Healthy & & & & & & & \\
\hline Positive & & & & & & & \\
\hline 2639 & 5.08 & -8 & -77 & 17 & L & Cuneus & 18 \\
\hline & 5.05 & -14 & -77 & 8 & L & Cuneus & 17 \\
\hline & 4.79 & -12 & -60 & 0 & L & Lingual gyrus & 18 \\
\hline 1472 & 5.10 & -18 & -91 & 26 & L & Cuneus & 18 \\
\hline & 5.09 & -6 & -95 & 19 & L & Cuneus & 18 \\
\hline 894 & 7.16 & 21 & -66 & 14 & R & Posterior cingulate & 31 \\
\hline & 4.35 & 11 & -62 & 12 & $\mathrm{R}$ & Posterior cingulate & 30 \\
\hline Negativ & led & & & & & & \\
\hline 1008 & 4.93 & 15 & -90 & -7 & R & Lingual gyrus & 17 \\
\hline & 3.97 & 14 & -98 & 1 & R & Cuneus & 17 \\
\hline 877 & 5.59 & -16 & -70 & 6 & L & Cuneus & 30 \\
\hline & 4.24 & -10 & -65 & 4 & L & Cuneus & 30 \\
\hline & 4.15 & -21 & -61 & 3 & L & Posterior cingulate & 30 \\
\hline
\end{tabular}

Positive $>$ Negative No cluster FEW $<0.05$

22a11DS patients

Positive $>$ Scrambled

55

Precuneus 7

$5.82 \quad 23$

$-60$

Precuneus

$5.59 \quad 29$

$-62$

Superior parietal lobule

Inferior Parietal Lobule

Superior parietal lobule

Precuneus

Superior parietal lobule

Postcentral

Postcentral

Postcentral

Lingual gyrus

Lingual gyrus

Lingual gyrus

Lingual gyrus

Lingual gyrus

Lingual gyrus

8

7

8

$\begin{array}{ll}5.54 & -13 \\ 5.45 & -7 \\ 4.15 & -7 \\ 4.13 & -7 \\ 3.82 & -3 \\ 5.47 & -30 \\ 4.80 & -24 \\ 4.62 & -22 \\ 4.55 & -33 \\ 4.24 & -25 \\ 3.94 & -17\end{array}$

$-79$ 
with 22q11DS showed atypical patterns of activation during the processing of social information compared to healthy controls. Specifically, we observed reduced brain activation in regions belonging to the default mode network (DMN). Indeed, the DMN encompasses the PFC as the anterior cingulate cortex (ACC) in its anterior part; laterally, it comprises the bilateral IPL and the medial temporal lobes whereas posteriorly it includes the posterior cingulate cortex (PCC) and the precuneus $[37,38]$. In the current study, we found hypoactivation of these regions (except for temporal lobes) during the perception of social information in individuals with 22q11DS compared to controls. The DMN is a restingstate network more activated in the absence of a cognitive task and has been well described as being implicated in various socio-cognitive processes such as selfreferential processing or theory of mind $[39,40]$. Nevertheless, recent literature established that there is an overlap between regions attributed to the DMN and regions activated during socio-cognitive tasks [41, 42]. In disorders characterized by social functioning deficits, such as schizophrenia or autism spectrum disorder (ASD), alterations of the DMN have been previously reported. The majority of studies conducted in schizophrenia points to a decreased functional connectivity of the DMN [43-47], whereas in ASD, altered functional connectivity of DMN regions has been reported during both rest [48-56] and during social tasks [57].

In patients with 22q11DS, alterations of resting state networks, including the DMN, have also been demonstrated using various methods. Whereas studies investigating functional connectivity of multiple resting state networks found hypoconnectivity of the DMN in individuals with 22q11DS [57-59], a multimodal approach revealed both structural and functional connectivity disruption of the DMN [60]. A few studies also examined the behavioural correlates of these alterations. In particular, associations between the strength of the DMN long-range connectivity and social functioning [58] and between a functional decrease of the DMN and prodromal symptom severity have been reported [57].

Our results are in accordance with previous literature in the 22q11DS population and demonstrate a hypoactivation of the DMN during the perception of social information, a basic socio-cognitive process. Taken together, these findings support the involvement of the DMN in socio-cognitive processes and point to a central role of this network in the pathophysiology of 22q11DS. The present study also suggests that alterations of this network could account for social dysfunctions observed in the syndrome. However, it should be noted that post hoc analyses did not reveal any significant correlations between activations within regions of the DMN and the socio-cognitive measures in the group of patients with
22q11DS. Because our sample size is relatively small and we used a non-direct measure of social functioning (parent-reported questionnaire), this finding should be interpreted carefully. Moreover, previous studies showing associations between social functioning and the DMN investigated functional connectivity within the DMN whereas the present study examined BOLD signal. Consequently, further studies investigating the functional connectivity during social perception as well as the association between impaired social perception network and social functioning are required.

Surprisingly, we did not find any significant difference between groups during social perception in the fusiform gyrus, while previous findings found reduced fusiform response during face perception in individuals with 22q11DS [8,9]. We also failed to see differences in the amygdala and superior temporal sulcus, whereas these two regions appear to be significantly activated during social vs. non-social perception in healthy controls [21]. The lack of result could be due to thresholding differences applied between studies. Indeed, while we applied very strict corrections, previous studies reported results with lower and less-sensitive thresholds [8, 9, 21]. Further studies investigating the activation of these regions during social perception are required.

Taken together, our results clearly indicate alterations in the social perception network in participants with 22q11DS. These alterations could lead to difficulties in social information processing, which could in turn influence various aspects of social cognition and thus contribute to social functioning impairments frequently observed in this syndrome [4-6].

Several explanations could be made to explain differences in the social perception network in 22q11DS. As proposed by Azuma et al. [9], one possible explanation may be a variation in dopamine, which is known to be a neuromodulator of socio-cognitive processes [61]. Individuals with 22q11DS are hemizygous for some genes, including the catechol-O-methyl transferase (COMT) gene that plays a role in dopamine degradation. Consequently, dopamine levels in regions of the social brain could be modified in 22q11DS [62,63] and lead to altered brain response during social processing. It has already been suggested that dopaminergic genetic variation impacts social perception and behaviour [64]. Indeed, some studies investigating the effects of dopamine variation on ventral striatum response to social reward have demonstrated that heightened dopamine signal is associated with increased response to social reward (impulsive or aggressive) [65, 66] and increased social approach behaviours. Future research investigating the impact of dopamine variation on social perception in 22q11DS is required. Alternatively, these results could also be driven by differences in brain anatomy. Indeed, 
a previous study conducted in participants with 22q11DS reported a positive relationship between fronto-striatal grey matter volume and social behavioural difficulties [67]. Moreover, structural changes in regions that are part of the social network, such as frontal regions, PCC, and ACC, have already been reported in the 22q11DS literature [68-70]. Thus, larger studies are required to examine the relationship between functional and structural alterations within the social network in the 22q11DS population.

Comparison of the neural correlates of emotion processing within groups revealed that positive stimuli perception was associated with increased activation in the lingual gyrus in both patients and controls. Conversely, increased activation to positive images was found in the PCC and cuneus in controls only, while increased activation in the precuneus, postcentral gyrus, and superior parietal lobule was observed solely in patients. During negative stimuli perception, controls showed greater activation of the right lingual gyrus, bilateral cuneus, and left PCC, while no significant activation was found in patients. Between-group comparisons did not reveal significant difference. These results suggest that during negative and positive emotion processing, patients and healthy controls present a similar pattern of activation. Thus, compared to the social perception network that was clearly atypical in 22q11DS, the neural correlates of emotion perception appear to be preserved in this population. Our results are in contradiction with previous work in the 22q11DS that described hypoactivation of regions related to emotional processing. Indeed, hypoactivation in frontal regions during negative emotional viewing $[8,9]$ and reduced activation of the insula during mixed emotional faces [7] was found. However, it should be noted that van Amelsvoort et al. reported results from a very small sample (eight patients versus nine controls), so that differences observed between the two studies should be interpreted with caution.

Finally, we examined the influence of emotions on social perception by investigating interactive processing of social content and valence. Our results did not reveal any significant social content $x$ valence interactions either within- or between-group comparisons. Those results are in contraction with previous studies conducted in healthy individuals where a social content $x$ valence interaction emerged [19-21]. Indeed, while Norris et al. [19] and Scharpf et al. [20] reported significant interactions in the thalamus, superior temporal sulcus, and middle orbito-temporal cortex, as well as in the anterior insula and lateral medial prefrontal cortex, Vrtička et al. [21] found such interaction within the amygdala, fusiform, anterior superior frontal gyrus, and middle occipital cortex. One explanation for this absence of social $x$ valence content interaction is a lack of statistical power due the sample size. However, it should be noted that the previous studies reported whole brain or region of interest results with an uncorrected threshold, while we used strict corrections by combining a primary voxellevel statistical threshold at $p<0.001$ (uncorrected, whole brain) and $k \geq 20$, with a cluster-extent familywise correction (FWEc <0.05). As no differences in interactive processing of social content and valence between groups were found, this suggests that social processing is impaired regardless of the valence in the 22q11DS population.

The results of the present study should be interpreted in light of several limitations. First, we investigated the neural correlates of social perception whereas it is well documented that patients with 22q11DS present visual perception and processing deficits [70, 71]. Indeed, difficulties in object, face, and emotion recognition have been described in the 22q11DS population [71]. Moreover, Magnée et al. [70] reported abnormal transmission between higher and lower visual cortex areas during the presentation of visual stimuli in an event-related potential study. As visual processing deficits could influence social perception, their impact on the observed findings should be further examined. Secondly, although the number of participants included in this study is higher compared to previous work in this field of research [7-9], our sample size remains relatively small. Consequently, results need to be interpreted carefully and studies including a larger sample are required. Thirdly, the neural correlates of social-emotional perception have only been investigated in a cross-sectional way. Thus, the time window during which functional alterations in the social perception network emerge remains unknown. Longitudinal studies examining the developmental trajectory of these alterations in 22q11DS are therefore required. An additional limitation is the potential influence of medication within the group of patients with 22q11DS, which was not investigated in the current study. However, we chose to exclude patients with schizophrenia or another psychotic disorder diagnosis, and only one patient was under antipsychotics at the time of testing. Finally, the neural correlates of additional socio-cognitive processes should be examined in further studies (e.g. theory of mind). Indeed, a better understanding of the neural correlates of social deficits in the 22q11DS population is required.

\section{Conclusion}

The present study provides evidence of specific alterations in the social perception network, irrespective of valence, in the 22q11DS population. Moreover, emotional processing of negative and positive stimuli seems preserved in individuals with 22q11DS. This is the first study investigating lower-order processes of social cognition in the 22q11DS population. The 
observed alterations are likely to influence higher order socio-cognitive processes. Deficits of social information processing could therefore be a key factor leading to socio-cognitive impairment in the 22q11DS population. Taken together, these findings highlight the need to better understand the emergence of social perception deficits in 22q11DS. Future studies investigating the developmental trajectory of social perception and its association with socio-cognitive processes are required.

\section{Additional file}

Additional file 1: Table S1. Characteristic of the IAPS images selected. (DOCX $15 \mathrm{~kb})$

\begin{abstract}
Abbreviations
22q11.2DS: 22q11 deletion syndrome; ACC: Anterior cingulate cortex; ASD: Autism spectrum disorder; BFRT: Benton Facial Recognition Test; BOLD: Blood-oxygenation-level-dependent; COMT: Catechol-O-methyl transferase; DARTEL: Diffeomorphic anatomical registration using exponential lie algebra algorithm; DMN: Default mode network; fMRI: functional magnetic resonance imaging; FWEC: Family-wise correction; GLM: General linear model; IAPS: International Affective Picture System; IPL: Inferior parietal lobule; KSADS-PL: Kiddie-Schedule for Affective Disorders and Schizophrenia Present and Lifetime version; MNI: Montreal Neurologic Institute; PCC: Posterior cingulate cortex; QF-PCR: quantitative fluorescent polymerase chain reaction; ROI: Region of interest; SCID-I: Structured Clinical Interview for DSM-IV axis disorders; SIPS: Structured Interview for Psychosis-risk Syndromes; SPM12: Statistical Parametric Mapping 12; SRS: Social Responsiveness Scale; VCFS: Velocardiofacial syndrome; WAIS III: Wechsler Adult Intelligence Scale, third version; WAIS IV: Wechsler Adult Intelligence Scale, fourth version; WISC III: Wechsler Intelligence Scale for Children, third version; WISC IV: Wechsler Intelligence Scale for Children, fourth version
\end{abstract}

\section{Acknowledgements}

The authors would like to thank all the families who contributed to the study as well as the family associations (Génération 22, Connect 22, Relais 22, and Creaf 22) for their ongoing support. Special thanks go to S. Menghetti, A. Zaharia, J. Maeder, and V. Pouillard from the Office Médico-Pédagogique Research Unit for their implication in the project in Geneva.

\section{Funding}

This work was supported by research grants from the Swiss National Science Foundation (grant no. 324730_144260) and The National Center of Competence in Research "Synapsy - The Synaptic Bases of Mental Diseases" to E.S (grant no. 51NF40-158776) and to M.D (grant no. 100014-135311/1).

\section{Availability of data and materials}

Not applicable.

\begin{abstract}
Authors' contributions
$M S, M D$, and PV designed the study. $L D, M S, M D, L C$, and SE acquired the data, including clinical assessments and cognitive testing. LD and MS analysed the data with the supervision of PV for the statistical analysis. LD and MS wrote the first draft of the manuscript; and all authors contributed for the interpretation of the results and the writing of the manuscript. All authors have approved the final manuscript.
\end{abstract}

\section{Ethics approval and consent to participate}

The authors assert that all procedures contributing to this work comply with the ethical standards of the relevant national and institutional committees on human experimentation and with the Helsinki Declaration of 1975, as revised in 2008.

\section{Consent for publication}

The authors declare that the manuscript did not contain individual person's data in any form.

\section{Competing interests}

The authors declare that they have no competing interests.

\section{Publisher's Note}

Springer Nature remains neutral with regard to jurisdictional claims in published maps and institutional affiliations.

\section{Author details}

${ }^{1}$ Developmental Imaging and Psychopathology Laboratory, Department of Psychiatry, School of Medicine, University of Geneva, Campus Biotech, Chemin des mines 9, 1202 Geneva, Switzerland. ${ }^{2}$ Center for Contextual Psychiatry, Department of Neurosciences, Research Group Psychiatry, KU Leuven, Leuven, Belgium. ${ }^{3}$ Department of Social Neuroscience, Max Planck Institute for Human Cognitive and Brain Sciences, Leipzig, Germany. ${ }^{4}$ Developmental Clinical Psychology Research Unit, Faculty of Psychology and Educational Sciences, University of Geneva, Geneva, Switzerland. ${ }^{5}$ Research Department of Clinical, Educational and Health Psychology, University College London, London, UK. ${ }^{6}$ Department of Genetic Medicine and Development, School of Medicine, University of Geneva, Geneva, Switzerland.

Received: 6 December 2017 Accepted: 23 March 2018

Published online: 10 April 2018

\section{References}

1. Shprintzen RJ. Velo-cardio-facial syndrome: 30 years of study. Dev Disabil Res Rev. 2008;14:3-10.

2. Grati FR, Molina Gomes D, Ferreira JCPB, et al. Prevalence of recurrent pathogenic microdeletions and microduplications in over 9500 pregnancies. Prenat Diagn. 2015;35:801-9.

3. Murphy KC. Annotation: velo-cardio-facial syndrome. J Child Psychol Psychiatry. 2005:46:563-71.

4. Swillen A, Devriendt K, Legius E, et al. The behavioural phenotype in velocardio-facial syndrome (VCFS): from infancy to adolescence. Genet Couns Geneva Switz. 1999;10:79-88.

5. Angkustsiri K, Goodlin-Jones B, Deprey L, et al. Social impairments in chromosome 22q11.2 deletion syndrome (22q11.2DS): autism spectrum disorder or a different endophenotype? J Autism Dev Disord. 2014;44:739-46.

6. Kiley-Brabeck K, Sobin C. Social skills and executive function deficits in children with the 22q11 deletion syndrome. Appl Neuropsychol. 2006;13: 258-68.

7. van Amelsvoort T, Schmitz N, Daly E, et al. Processing facial emotions in adults with velo-cardio-facial syndrome: functional magnetic resonance imaging. Br J Psychiatry J Ment Sci. 2006;189:560-1.

8. Andersson F, Glaser B, Spiridon M, et al. Impaired activation of face processing networks revealed by functional magnetic resonance imaging in 22q11.2 deletion syndrome. Biol Psychiatry. 2008;63:49-57.

9. Azuma R, Deeley Q, Campbell LE, et al. An fMRI study of facial emotion processing in children and adolescents with 22q11.2 deletion syndrome. J Neurodev Disord. 2015;7:1.

10. Atkinson AP, Adolphs R. The neuropsychology of face perception: beyond simple dissociations and functional selectivity. Philos Trans R Soc B Biol Sci. 2011;366:1726-38.

11. Haxby JV, Hoffman EA, Gobbini MI. Human neural systems for face recognition and social communication. Biol Psychiatry. 2002;51:59-67.

12. Vuilleumier $P$, Pourtois $G$. Distributed and interactive brain mechanisms during emotion face perception: evidence from functional neuroimaging. Neuropsychologia. 2007:45:174-94.

13. Sabatinelli D, Fortune EE, Li Q, et al. Emotional perception: meta-analyses of face and natural scene processing. Neurolmage. 2011;54:2524-33.

14. Bjorkquist OA, Herbener ES. Social perception in schizophrenia: evidence of temporo-occipital and prefrontal dysfunction. Psychiatry Res. 2013;212:175-82.

15. Campbell LE, Stevens AF, McCabe K, et al. Is theory of mind related to social dysfunction and emotional problems in 22q11.2 deletion syndrome (velocardio-facial syndrome)? J Neurodev Disord. 2011;3:152-61. 
16. Campbell LE, McCabe KL, Melville $J$, et al. Social cognition dysfunction in adolescents with 22q11.2 deletion syndrome (velo-cardio-facial syndrome): relationship with executive functioning and social competence/functioning. J Intellect Disabil Res. 2015;59:845-59.

17. Kober H, Barrett LF, Joseph J, et al. Functional grouping and corticalsubcortical interactions in emotion: a meta-analysis of neuroimaging studies. Neurolmage. 2008;42:998-1031.

18. Satpute $A B$, Kang J, Bickart KC, et al. Involvement of sensory regions in affective experience: a meta-analysis. Front Psychol. 2015;6 https://doi.org/ 10.3389/fpsyg.2015.01860.

19. Norris CJ, Chen EE, Zhu DC, et al. The interaction of social and emotional processes in the brain. J Cogn Neurosci. 2004;16:1818-29.

20. Scharpf KR, Wendt J, Lotze M, et al. The brain's relevance detection network operates independently of stimulus modality. Behav Brain Res. 2010;210:16-23.

21. Vrtička P, Sander D, Vuilleumier P. Lateralized interactive social content and valence processing within the human amygdala. Front Hum Neurosci. 2013;6 https://doi.org/10.3389/fnhum.2012.00358.

22. Reich W. Diagnostic interview for children and adolescents (DICA). J Am Acad Child Adolesc Psychiatry. 2000;39:59-66.

23. Kaufman J, Birmaher B, Brent D, et al. Schedule for affective disorders and schizophrenia for school-age children-present and lifetime version (K-SADS-PL): initial reliability and validity data. J Am Acad Child Adolesc Psychiatry. 1997;36:980-8.

24. First M, Gibbon M, Spitzer R, Williams J, Benjamin L. Structured Clinical Interview for the DSM-IV Axis I Disorders (SCID-I). Washington: American Psychiatric Association; 1996.

25. Miller TJ, McGlashan TH, Rosen $J$, et al. Prodromal assessment with the structured interview for prodromal syndromes and the scale of prodromal symptoms: predictive validity, interrater reliability, and training to reliability Schizophr Bull. 2003;29:703-15.

26. Wechsler D. The Wechsler Intelligence Scale for Children, 3rd edn. San Antonio: The Psychological Corporation; 1991.

27. Weschsler D. Wechsler: WISC-IV: Echelle d'Intelligence de Wechsler. (4e ed.) Paris: (2005, accessed 28 Mar 2017).

28. Wechsler D. Wechsler Adult Intelligence Scale (WAIS-3), 3rd edn. San Antonio: The Psychological Corporation; 1997.

29. Weschsler D. Weschsler adult intelligence scale-fourth edition (WAIS IV). San Antonio. (2008, accessed 27 Mar 2017)

30. Benton AL, Sivan AB, des Hamsher $K$, et al. Contributions to neuropsychological assessment: a clinical manual. 2 edition. New York: Oxford University Press; 1994.

31. Constantino J. (SRS $\left.{ }^{T M}-2\right)$ social responsiveness scale $e^{T M}$, second edition (2012, accessed 8 Mar 2018).

32. Lang PJ, Bradley MM, Cuthbert BN. Technical report A-6, international affective picture system (IAPS): digitized photographs, instruction manual and affective ratings. Gainesville: University of Florida; 2005

33. Telegraphics - Free plugins for Photoshop \& Illustrator...and other software. http://www.telegraphics.com.au/sw/product/Scramble (accessed 10 Nov 2017)

34. MNI2Tal > Biolmage Suite | Bioimaging Sciences | Yale School of Medicine. http://bioimagesuite.yale.edu/mni2tal/ (accessed 10 Nov 2017).

35. Vrtička $P$, Sander D, Vuilleumier P. Effects of emotion regulation strategy on brain responses to the valence and social content of visual scenes. Neuropsychologia. 2011;49:1067-82.

36. MarsBaR region of interest toolbox for SPM - MarsBaR 0.44 documentation. http://marsbar.sourceforge.net/ (accessed 10 Nov 2017).

37. Raichle ME, MacLeod AM, Snyder AZ, et al. A default mode of brain function. Proc Natl Acad Sci U S A. 2001;98:676-82.

38. Rosazza C, Minati L. Resting-state brain networks: literature review and clinical applications. Neurol Sci Off J Ital Neurol Soc Ital Soc Clin Neurophysiol. 2011;32:773-85.

39. Spreng RN, Mar RA, Kim ASN. The common neural basis of autobiographical memory, prospection, navigation, theory of mind, and the default mode: a quantitative meta-analysis. J Cogn Neurosci. 2009;21:489-510.

40. Grecucci A, Giorgetta C, Bonini N, et al. Reappraising social emotions: the role of inferior frontal gyrus, temporo-parietal junction and insula in interpersonal emotion regulation. Front Hum Neurosci. 2013;7 https:/doi.org/10.3389/ fnhum.2013.00523.

41. Schilbach L, Eickhoff SB, Rotarska-Jagiela A, et al. Minds at rest? Social cognition as the default mode of cognizing and its putative relationship to the 'default system' of the brain. Conscious Cogn. 2008;17:457-67.
42. Mars RB, Neubert F-X, Noonan MP, et al. On the relationship between the "default mode network" and the "social brain". Front Hum Neurosci. 2012;6 https://doi.org/10.3389/fnhum.2012.00189.

43. Buckner RL, Andrews-Hanna JR, Schacter DL. The brain's default network: anatomy, function, and relevance to disease. Ann N Y Acad Sci. 2008;1124:1-38.

44. Narr KL, Leaver AM. Connectome and schizophrenia. Curr Opin Psychiatry. 2015;28:229-35

45. Jafri MJ, Pearlson GD, Stevens $M$, et al. A method for functional network connectivity among spatially independent resting-state components in schizophrenia. Neurolmage. 2008;39:1666-81.

46. Greicius MD, Krasnow B, Reiss AL, et al. Functional connectivity in the resting brain: a network analysis of the default mode hypothesis. Proc Natl Acad Sci U S A. 2003;100:253-8.

47. Lynall M-E, Bassett DS, Kerwin R, et al. Functional connectivity and brain networks in schizophrenia. J Neurosci. 2010;30:9477-87.

48. Cherkassky VL, Kana RK, Keller TA, et al. Functional connectivity in a baseline resting-state network in autism. Neuroreport. 2006;17:1687-90.

49. Kennedy DP, Courchesne E. The intrinsic functional organization of the brain is altered in autism. Neurolmage. 2008;39:1877-85.

50. Monk CS, Peltier SJ, Wiggins JL, et al. Abnormalities of intrinsic functional connectivity in autism spectrum disorders. Neurolmage. 2009;47:764-72.

51. Weng S-J, Wiggins $J$, Peltier SJ, et al. Alterations of resting state functional connectivity in the default network in adolescents with autism spectrum disorders. Brain Res. 2010;1313:202-14.

52. Assaf $M$, Jagannathan $\mathrm{K}$, Calhoun VD, et al. Abnormal functional connectivity of default mode sub-networks in autism spectrum disorder patients. Neurolmage. 2010;53:247-56.

53. Lynch CJ, Uddin LQ, Supekar K, et al. Default mode network in childhood autism: posteromedial cortex heterogeneity and relationship with social deficits. Biol Psychiatry. 2013;74:212-9.

54. von dem Hagen EAH, Stoyanova RS, Baron-Cohen S, et al. Reduced functional connectivity within and between 'social' resting state networks in autism spectrum conditions. Soc Cogn Affect Neurosci. 2013;8:694-701.

55. Redcay E, Moran JM, Mavros PL, et al. Intrinsic functional network organization in high-functioning adolescents with autism spectrum disorder. Front Hum Neurosci. 2013;7 https://doi.org/10.3389/fnhum.2013.00573.

56. Moseley RL, Ypma RJF, Holt RJ, et al. Whole-brain functional hypoconnectivity as an endophenotype of autism in adolescents. Neurolmage Clin. 2015;9:140-52.

57. Schreiner MJ, Karlsgodt KH, Uddin LQ, et al. Default mode network connectivity and reciprocal social behavior in 22q11.2 deletion syndrome. Soc Cogn Affect Neurosci. 2014;9:1261-7.

58. Debbané M, Lazouret $M$, Lagioia $A$, et al. Resting-state networks in adolescents with 22q11.2 deletion syndrome: associations with prodromal symptoms and executive functions. Schizophr Res. 2012;139:33-9.

59. Mattiaccio LM, Coman IL, Schreiner MJ, et al. Atypical functional connectivity in resting-state networks of individuals with 22q11.2 deletion syndrome: associations with neurocognitive and psychiatric functioning. Neurodev Disord. 2016;8:2

60. Padula MC, Schaer M, Scariati E, et al. Structural and functional connectivity in the default mode network in 22q11.2 deletion syndrome. J Neurodev Disord. 2015;7:23.

61. Berton O, McClung CA, Dileone RJ, et al. Essential role of BDNF in the mesolimbic dopamine pathway in social defeat stress. Science. 2006;311:864-8.

62. Fallgatter AJ, Lesch KP. 22q11.2 deletion syndrome as a natural model for COMT haploinsufficiency-related dopaminergic dysfunction in ADHD. Int J Neuropsychopharmacol. 2007;10:295-9.

63. Gothelf D, Hoeft F, Hinard C, et al. Abnormal cortical activation during response inhibition in 22q11.2 deletion syndrome. Hum Brain Mapp. 2007; 28:533-42.

64. Skuse DH, Gallagher L. Genetic influences on social cognition. Pediatr Res. 2011;69:85R-91R.

65. Forbes EE, Brown SM, Kimak M, et al. Genetic variation in components of dopamine neurotransmission impacts ventral striatal reactivity associated with impulsivity. Mol Psychiatry. 2009;14:60-70.

66. Seo D, Patrick CJ, Kennealy PJ. Role of serotonin and dopamine system interactions in the neurobiology of impulsive aggression and its comorbidity with other clinical disorders. Aggress Violent Behav. 2008;13:383-95.

67. Campbell LE, Daly E, Toal F, et al. Brain and behaviour in children with 22q11.2 deletion syndrome: a volumetric and voxel-based morphometry MRI study. Brain J Neurol. 2006;129:1218-28. 
68. Shashi V, Veerapandiyan A, Schoch K, et al. Social skills and associated psychopathology in children with chromosome 22q11.2 deletion syndrome: implications for interventions. J Intellect Disabil Res. 2012;56:865-78.

69. Simon TJ, Ding L, Bish JP, et al. Volumetric, connective, and morphologic changes in the brains of children with chromosome 22q11.2 deletion syndrome: an integrative study. Neurolmage. 2005;25:169-80.

70. Magnée MJCM, Lamme VAF, de Sain-van der Velden MGM, et al. Proline and COMT status affect visual connectivity in children with 22q11.2 deletion syndrome. PLoS One. 2011;6:e25882.

71. McCabe KL, Marlin S, Cooper G, et al. Visual perception and processing in children with 22a11.2 deletion syndrome: associations with social cognition measures of face identity and emotion recognition. J Neurodev Disord. 2016;8 https://doi.org/10.1186/s11689-016-9164-7.

Submit your next manuscript to BioMed Central and we will help you at every step:

- We accept pre-submission inquiries

- Our selector tool helps you to find the most relevant journal

- We provide round the clock customer support

- Convenient online submission

- Thorough peer review

- Inclusion in PubMed and all major indexing services

- Maximum visibility for your research

Submit your manuscript at www.biomedcentral.com/submit
Biomed Central 